\title{
NOUVELle
}

\section{Ouvrir le cerveau à de nouvelles thérapies}

Ludovic Collin
F. Hoffmann-La Roche Ltd ; Preclinical Research Neuroscience, PCDMR ; Grenzacherstrasse, CH-4070 Basel, Suisse.

ludovic.collin@roche.com
La barrière hémato-encéphalique

La paroi de ces capillaires forme une barrière physiologique: la barrière hémato-encéphalique, qui sépare le système nerveux central de la circulation sanguine. Cette barrière régule l'homéostasie du cerveau en protégeant les cellules cérébrales des variations de concentrations d'hormones et de neurotransmetteurs, et des modifications de l'acidité (ou $\mathrm{pH})$ du sang. Elle prévient également l'entrée de substances toxiques et de microorganismes pathogènes dans le cerveau et la moelle épinière.

La barrière hémato-encéphalique est constituée essentiellement de trois types cellulaires. Les cellules endothéliales polarisées, qui forment la paroi des vaisseaux sanguins (la face luminale de ces cellules étant en contact avec la circulation sanguine), sont entourées par une couche protéique de 40 à $50 \mathrm{~nm}$ d'épaisseur appelée lame basale. Elles sont liées par des jonctions serrées qui rendent imperméable l'espace entre les

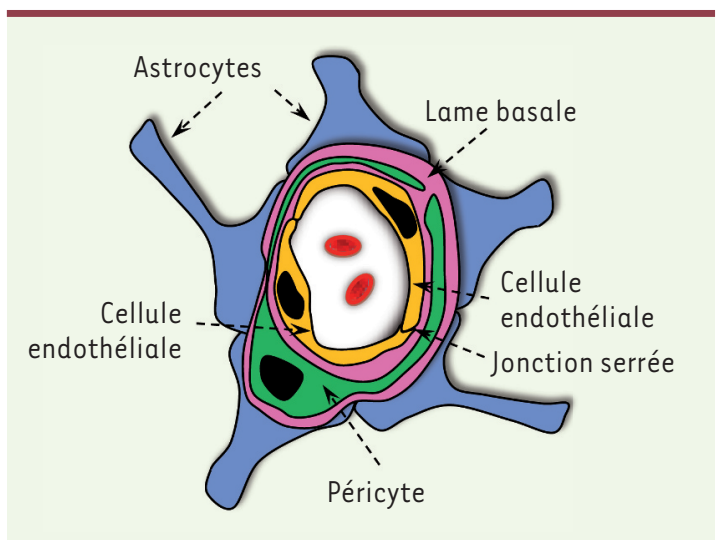

Figure 1. Représentation schématique de la barrière hématoencéphalique à l'échelle cellulaire. cellules. Les péricytes et les astrocytes sont les deux autres types cellulaires qui forment la barrière hémato-encéphalique cellulaire (Figure 1) [1]. Les péricytes sont intégrés dans la lame basale, alors que les astrocytes interagissent avec chaque microvaisseau. Les péricytes et les astrocytes sont importants pour la croissance et la fonction de la barrière hémato-encéphalique [2].

Malgré son étanchéité, cette barrière doit assurer le transport de nutriments et d'oxygène vers le cerveau ainsi que l'élimination des produits toxiques issus du métabolisme. Ces échanges se font par l'intermédiaire de différents mécanismes. Certaines petites molécules lipidiques peuvent entrer dans le cerveau par transport passif. Des transporteurs spécialisés sont aussi présents sur la membrane des cellules endothéliales et facilitent le passage de certaines macromolécules. C'est le cas de molécules vitales comme le glucose, les acides aminés et les nucléosides. D'autres molécules contenues dans le sang, comme l'insuline et la transferrine, utilisent des récepteurs spécifiques et le mécanisme de transcytose pour accéder au tissu nerveux [1]. La transcytose est un mécanisme cellulaire qui a lieu dans des cellules polarisées. Il permet le passage de molécules du domaine apical au domaine basal des cellules. Au niveau du système vasculaire cérébral, la transferrine interagit avec son récep- teur (TfR) qui est situé sur la face luminale des cellules endothéliales. Le complexe transferrine-TfR formé est alors internalisé, un mécanisme appelé endocytose. Ce processus d'endocytose implique que la membrane plasmique s'invagine pour entourer le récepteur et son ligand et ainsi former des structures vésiculaires intracellulaires. Les vésicules traversent ensuite le cytoplasme pour atteindre la face opposée de la cellule ou elles fusionnent avec la membrane plasmique et libèrent la transferrine dans le tissu nerveux (Figure 2).

\section{Une navette efficace pour traverser} la barrière hémato-encéphalique Ces dernières années, de nombreuses molécules thérapeutiques ont été proposées pour traiter les maladies neurodégénératives; elles sont basées sur l'utilisation de grosses molécules (anticorps monoclonaux, peptides, etc.) [3]. Des études précliniques ont prouvé l'efficacité de telles molécule, qui peuvent ralentir la progression de ces maladies et cela malgré leur pénétration limitée dans le cerveau. En effet, Banks et al. ont par exemple montré que seulement $0,1 \%$ des anticorps injectés par voie intraveineuse traversent la barrière hémato-encéphalique pour atteindre leur cible dans le tissu nerveux de la souris [4].

De nombreux travaux ont été entrepris afin d'établir de nouvelles stratégies pour augmenter le passage de grosses molécules thérapeutiques au travers de la barrière hémato-encéphalique. Ces approches, qui incluent le développement et I'utilisation de nanoparticules, de 
A

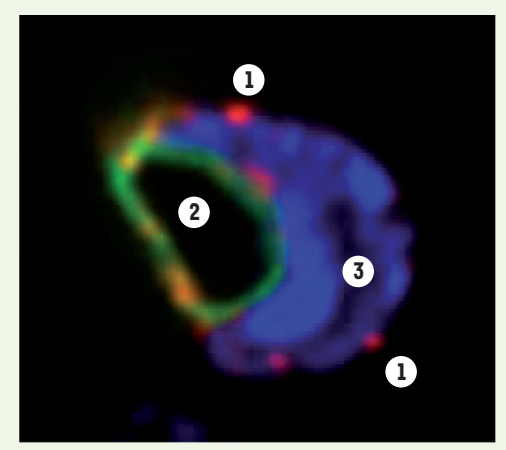

1 Brain shuttle

2 Lumière du capillaire

3 Noyau d'une cellule

endothéliale
B
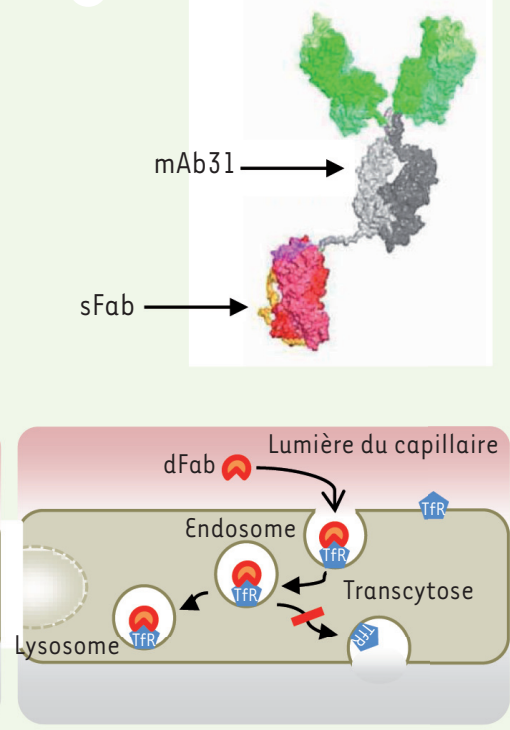

Figure 2. Le brain shuttle et son mode de fonctionnement. A. Marquage immunofluorescent montrant le brain shuttle (rouge) traversant un microcapillaire du cerveau. B. Représentation schématique du brain shuttle combiné à l'anticorps mAb31. C. Modèle simplifié expliquant le mécanisme de transcytose du brain shuttle (sFab : molécule monovalente; dFab : molécule bivalente).

liposomes, de peptides, de virus, ou d'anticorps, ont connu des fortunes diverses [3].

La stratégie employée par Hoffmann-La Roche a été de cibler le TfR, récepteur naturellement exprimé par les cellules endothéliales des capillaires sanguins, et le mécanisme de transcytose [5]. Le système que nous avons développé est basé sur un fragment Fab monovalent d'un anticorps dirigé contre le TfR. L'interaction de ce module (anti-TfR Fab), appelé brain shuttle, avec le TfR est suffisante pour induire le phénomène de transcytose et n'empêche pas la transferrine d'interagir avec son récepteur. Des images de microscopie confocale à haute résolution montrent la présence de vésicules contenant le brain shuttle traversant les microcapillaires 30 minutes seulement après l'administration de ce dernier chez la souris (Figure 2). Afin d'évaluer le potentiel thérapeutique du brain shuttle, le

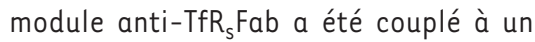
anticorps monoclonal, mAb3l, dirigé contre le peptide d'amyloïde bêta $(A \beta)$ (Figure 2) [6]. A $\beta$ est le peptide toxique qui s'accumule et forme les plaques amyloïdes dans le cerveau de patients atteints de la maladie d'Alzheimer [7]. Des études précliniques utilisant cet anticorps ont montré son efficacité pour éliminer les plaques amyloïdes dans le modèle de souris transgénique PS2APP, modèle murin de la maladie d'Alzheimer [6]. Nous montrons dans notre étude et dans ce même modèle animal, que les premières plaques amyloïdes sont détectées par la construction anti-TfR $\mathrm{R}_{\mathrm{s}}$ Fab-mAb3l seulement huit heures après son administration en périphérie par voie intraveineuse. Cela montre également que ni le couplage de l'anticorps mAb3l au brain shuttle, ni la transcytose de la construction n'affectent la reconnaissance par l'anticorps de sa cible. Cependant, lorsque mAb31 est combiné a un brain shuttle formé de deux fragments Fab contre le TfR (anti-TfR Fab, molécule bivalente), cette construction ne peut traverser la barrière hématoencéphalique même lorsque les animaux sont analysés sept jours après administration de la construction. Nous avons montré que la molécule bivalente est endocytée par les cellules endothéliales puis ciblée aux lysosomes, des organites cellulaires ayant pour fonction la digestion de macromolécules, mais elle n'est pas libérée dans le cerveau comme l'est la molécule monovalente (Figure 2). La molécule bivalente, ainsi que les TfR avec lesquels elle interagit, est alors dégradée par les enzymes lysosomales, réduisant ainsi l'expression du TfR sur la membrane des cellules endothéliales. Des études complémentaires sont actuellement en cours afin d'élucider les mécanismes moléculaires régulant la différence de trafic des constructions monovalente et bivalente. Nos résultats montrent donc que la valence du brain shuttle (nombre de modules interagissant avec le TfR) est cruciale pour un passage efficace de la construction de la circulation sanguine vers le parenchyme cérébral.

L'utilisation du brain shuttle nous a permis d'augmenter 55 fois la pénétration de notre anticorps anti-A $\beta$ dans le cerveau en comparaison de la molécule parentale (sans brain shuttle) et cela sans affecter l'intégrité de la barrière hémato-encéphalique. Ces résultats 
furent obtenus en utilisant les souris transgéniques PS2APP et en quantifiant le signal fluorescent résultant de l'interaction de l'anticorps mAb3l avec les plaques amyloïdes contenues dans le cerveau de ces souris [6]. Nous avons également montré, en utilisant le même modèle animal lors d'une étude prolongée quatre mois, que cette meilleure pénétration de l'anticorps est corrélée à une meilleure efficacité du traitement mesurée par l'augmentation de la dégradation des plaques amyloïdes.

\section{Perspectives}

Le brain shuttle que nous avons développé est basé sur l'utilisation d'un mécanisme de transport naturellement existant. Cette technologie pourrait offrir de nombreuses perspectives thérapeutiques pour le traitement de maladies neurologiques en permettant le passage de grosses molécules telles que des anticorps, des peptides, des enzymes ou autres facteurs de croissance dans le parenchyme cérébral. L'étude effectuée chez la souris devra maintenant être reproduite chez I'homme afin d'ouvrir les portes du cerveau à une nouvelle génération de molécules thérapeutiques. $\diamond$

\section{Opening the brain to new therapies}

\section{LIENS D’INTÉRÊT}

L'auteur déclare avoir des liens durables avec l'entreprise Hoffmann La Roche AG.

\section{RéFÉRENCES}

1. Zlokovic BV. The blood-brain barrier in health and chronic neurodegenerative disorders. Neuron 2008 ; 57: 178-201

2. Armulik A, Genove G, Mae M, et al. Pericytes regulate the blood-brain barrier. Nature 2010 ; 468: 557-561.

3. Pardridge WM. Drug transport across the bloodbrain barrier. J Cereb Blood Flow Metab 2012 ; 32 : 1959-72.

4. Banks WA, Terrell B, Farr SA, et al. Passage of amyloid beta protein antibody across the blood-brain barrier in a mouse model of Alzheimer's disease. Peptides $2002 ; 23: 2223-6$.

5. Niewoehner J, Bohrmann B, Collin L, et al. Increased brain penetration and potency of a therapeutic antibody using a monovalent molecular shuttle. Neuron $2014 ; 81: 49-60$.

6. Bohrmann B, Baumann K, Benz J, et al. Gantenerumab: a novel human anti-Abeta antibody demonstrates sustained cerebral amyloid-beta binding and elicits cell-mediated removal of human amyloid-beta. J Alzheimers Dis $2012 ; 28: 49-69$

7. Gosselet F, Candela P, Cecchelli R, Fenart L. La barrière hémato-encéphalique. Med Sci (Paris) 2011 ; 27 : 987-92.

\section{NOUVELL $\varepsilon$}

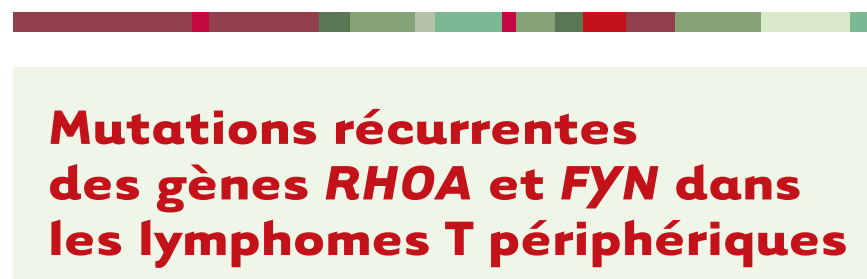

Lucile Couronné $^{1,2,3}$, Christian Bastard ${ }^{4}$, Olivier A. Bernard ${ }^{5,6,7}$

\section{Séquençage à haut débit dans} les lymphomes T périphériques Les lymphomes $T$ périphériques constituent un groupe hétérogène d'entités au pronostic globalement sombre dont les mécanismes de développement sont encore mal compris. Des mutations des gènes TET2 (methylcytosine dioxygenase 2), DNMT3A (DNA [cytosine-5-]methyltransferase 3 alpha) et IDH2 (isocitrate dehydrogenase), entraînant une dérégulation du contrôle de la méthylation de l'ADN, ont déjà été rapportées dans les deux sous-types histologiques les plus fréquents, le lymphome $T$ angioimmunoblastique (AITL) et le lymphome T périphérique, non spécifié (PTCL, NOS)
[1-4]. Les mutations de TET2 sont associées au phénotype T follicular helper (TFH) [2], défini par la positivité des cellules tumorales pour les antigènes CD10 et PDI, la prolifération de cellules folliculaires dendritiques positives pour le $C D 21$, et la présence de cellules $B$ exprimant l'EBV (Epstein Barr virus) [5], mais ne sont observées que chez une fraction des patients.

Deux groupes viennent de rapporter les résultats de séquençage à haut débit de lymphomes $T$ périphériques $[5,6]$. De façon similaire, après une première analyse par séquençage de l'ensemble des régions codantes du génome (whole-exome sequencing) des
${ }^{1}$ Service d'hématologie adulte, Assistance publique-Hôpitaux de Paris (APHP), hôpital Necker, Paris, France ;

2 Inserm UMR1163, CNRS ERL 8254, Institut Imagine, Paris, France ;

${ }^{3}$ Université Paris Descartes-Sorbonne Paris Cité, Paris, France ;

${ }^{4}$ Inserm U918, Université de Rouen, Centre Henri Becquerel, Rouen, France;

${ }^{5}$ Inserm U985, Villejuif, France ;

${ }^{6}$ Université Paris-Sud, Orsay, France ;

${ }^{7}$ Institut Gustave Roussy, Villejuif, France. lucile.couronne@gmail.com

cellules d'une série limitée de patients, les gènes mutés ont été analysés dans une cohorte d'extension indépendante de lymphomes T périphériques (159 patients pour l'équipe japonaise, 125 patients pour l'équipe américaine) en utilisant des techniques sensibles de détection comme le séquençage haut débit $[5,6]$ ou la PCR quantitative spécifique d'allèle [6].

Le reséquençage ciblé réalisé par l'équipe japonaise a confirmé la présence de mutations des gènes TET2, 